\title{
TRANSPARENCIA EN LAS INSTITUCIONES DE EDUCACIÓN SUPERIOR EN COLOMBIA
}

\section{Transparency in higher education institutions in Colombia}

\author{
Elías Said-Hung, María-Pilar Cousido-González e Inmaculada Berlanga- \\ Fernández
}

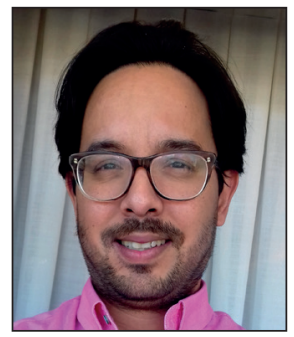

Elías Said-Hung es sociólogo y doctor en Ciencias de la Información. Profesor titular de la Facultad de Educación de la Universidad Internacional de la Rioja (UNIR), es miembro del grupo de investigación Procomm (Prospectivas en Comunicación Multimedia), de UNIR Research y consultor externo en Con-Tacto Humano.

http://orcid.org/0000-0002-0594-5906

Universidad Internacional de la Rioja Almansa, 101. 28040 Madrid, España esaidh@gmail.com

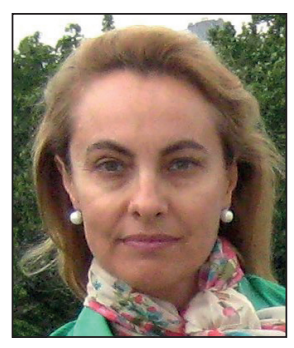

María-Pilar Cousido-González, licenciada en Derecho y doctora en Ciencias de la Información, es profesora titular del Departamento de Derecho Constitucional de la Facultad de Ciencias de la Información de la Universidad Complutense de Madrid (UCM). Es directora de la revista Derecom y del grupo de investigación Tra.Bu.Com. http://orcid.org/0000-0002-4828-5901

Universidad Complutense de Madrid, Facultad de Ciencias de la Información Avda. Complutense, s/n. Ciudad Universitaria 28040 Madrid, España cousidopilar@ccinf.ucm.es

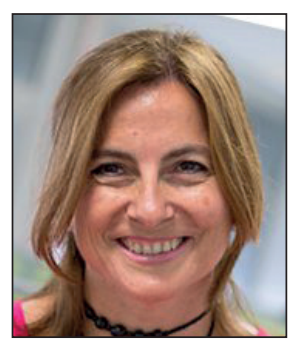

Inmaculada Berlanga-Fernández, licenciada y doctora en Filología, y licenciada y doctora en Comunicación Audiovisual por la Universidad de Granada, es profesora titular en la Universidad Internacional de la Rioja (UNIR). Visiting profesor en la Università Cattolica del Sacro Cuore de Milán. Es directora de Procomm (Prospectivas en Comunicación Multimedia) de UNIR Research. http://orcid.org/0000-0002-0135-624X

Universidad Internacional de la Rioja Almansa, 101. 28040 Madrid, España inmaculada.berlanga@unir.net

\section{Resumen}

Se analiza el nivel de transparencia de las instituciones de educación superior en Colombia transcurridos dos años desde la publicación de la Ley de transparencia y derecho de acceso a la información del país. El estudio recoge una muestra de 197 instituciones de educación superior pública y privada, registradas en el Sistema Nacional de Información de Educación Superior del Ministerio de Educación Nacional de Colombia en mayo de 2015. Los datos sobre la transparencia formal y la transparencia sustantiva de la información volcada en las webs de estas instituciones muestran que los niveles de transparencia son bajos y carecen de avances significativos. Los resultados revelan dificultades de aplicación efectiva de conceptos y procesos relacionados con la cibercultura.

\section{Palabras clave}

Instituciones de educación superior; Universidades; Internet; Sitios web; Webs; Transparencia; Visibilidad; Datos abiertos; Rendición de cuentas.

\section{Abstract}

The level of transparency in higher education institutions in Colombia two years after the enactment of the Law $1712 / 2014$ on Transparency and the right of access to public information in that country is analyzed. The study includes a sample of 197 public and private higher education institutions registered in the National Higher Education Information System of the 
Colombian Ministry of National Education in May 2015. Data observed on the websites of these institutions show a context where levels of formal and substantive transparency are low and no significant progress. The results reveal difficulties in the effective application of concepts and processes related to cyberculture.

\section{Keywords}

Higher education institutions; Universities; Internet; Websites; Transparency; Visibility; Open data; Accountability.

Said-Hung, Elías; Cousido-González, María-Pilar; Berlanga-Fernández, Inmaculada (2018). “Transparencia en las instituciones de educación superior en Colombia". El profesional de la información, v. 27, n. 1, pp. 162-171.

https://doi.org/10.3145/epi.2018.ene.15

\section{Introducción}

En los últimos años se han generado conceptos como datos masivos (big data), datos abiertos, datos enlazados y compartición de datos (data sharing) entre otros, que han facilitado la creciente promoción de la cultura digital y de la transparencia en las instituciones (Prince; Jolías, 2013; Hernández-Pérez; García-Moreno, 2013; Ferrer-Sapena; Sánchez-Pérez, 2013).

Estos conceptos se enmarcan en el movimiento open, surgido con la evolución de internet -especialmente de la web 2.0-, y que permite el aumento de la transparencia de los procesos que llevan a cabo los individuos (p. ej., investigadores) y las entidades públicas o privadas (Ferrer-Sapena; Sánchez-Pérez, 2013). Yu y Robinson (2012) describen un nuevo paradigma basado en la idea de que los datos creados por las administraciones públicas son propiedad de la sociedad en general.

El movimiento open y sus conceptos relacionados son controvertidos. Autores como Scassa y Campbell (2009), Farrel (2012) y Bates (2012) entre otros, plantean aspectos críticos en el control de los datos en abierto, los problemas de protección de datos para unos determinados fines y la reutilización de éstos para otros fines no contemplados inicialmente; también en el aprovechamiento potencial de este fenómeno en la generación de nuevos mercados de información en el sector público, sin fines de lucro. Otros autores (Johnson, 2012; Noveck, 2012; Yglesias, 2012) recogen los potenciales beneficios en las organizaciones para la resolución de problemas sociales y el aumento de la productividad en sectores profesionales vinculados a la comunicación ( $p$. ej., en el periodismo).

Pese al antagonismo del debate sobre el movimiento open en la actualidad, guardamos cercanía a los planteamientos de Lee (2012), Farrel (2012) y Gewin (2016), y reconocemos los retos en favor de la democracia con la promoción de la cultura de la transparencia por actores públicos y privados.

Como subrayan Lathrop y Ruma (2010), García-Macho (2010) y Sommerman (2010), en el entorno social marcado por este movimiento, los ciudadanos tienen derecho a controlar y participar en el acontecer de sus sociedades y en el devenir de las instituciones (públicas) que los representan, a través del acceso, reutilización y redistribución de los datos por cualquier persona.

Además, en palabras de Manfredi-Sánchez (2017), el desarrollo instrumental de la transparencia opera como me- canismo preventivo de la corrupción. También supone un potencial aumento de las oportunidades de mayor inteligencia organizacional por parte de las entidades privadas (Newman, 2012), que pueden verse beneficiadas económicamente y en su prestigio institucional a través de la exposición de sus datos e informaciones de forma abierta (Bernal, 2012), circunstancia que repercute en el acercamiento de los ciudadanos a nivel público y privado (Ferrer-Sapena; Sánchez-Pérez, 2013).

El impacto transformador ocasionado por la web 2.0 en las instituciones requiere la revisión e implantación de nuevas dinámicas de funcionamiento y sistemas estandarizados que ayuden a la promoción de la cibercultura actual y la transparencia de su funcionamiento. Así se facilita el acceso libre y compartido de contenidos generados o relacionados con diferentes instituciones actuales. Es el caso de las administraciones locales (Venkatesh et al., 2016; Rebolledo; Zamora-Medina; Rodríguez-Virgili, 2017); y de las universidades, donde autores como Downes (2001), Anderson, Boyles y Rainie (2012), Mengual-Andrés (2013), Roig, Mengual-Andrés y Suárez-Guerrero (2014) y Connaway (2014) reconocen estas ventajas en el ámbito educativo.

Tíscar Lara (2009) identificó a la universidad como agente activo en la cultura digital, cultura que construye y promueve contextos flexibles para facilitar el trabajo colaborativo en red y fomentar innovación creativa desde fuera de sus límites organizativos. Es una transformación que incluye la forma en que se muestra la información en los canales web 2.0 institucionales, y las diferentes estructuras y procedimientos donde se produce y divulga el conocimiento generado. Desde estos escenarios digitales se garantiza la información necesaria para conocer el funcionamiento y quehacer institucional. Montoya-Santiago (2016) precisa desde el ámbito jurídico las funciones y competencias de una oficina y de un portal de transparencia en una universidad pública.

El tema comporta un profundo debate académico y la revisión, ajuste y aplicación de marcos normativos que garanticen el acceso a la información, al menos desde el sector público. Es el caso de la Ley 1712/2014 en Colombia (Colombia, 2014), para promover la transparencia y rendición de cuentas de las instituciones públicas y las instituciones de educación superior del país. La Ley regula el derecho del acceso a la información pública y el establecimiento de un conjunto de procedimientos que lo garantice, y tiene en cuenta ciertas excepciones vinculadas con la publicidad de 
Tabla 1. Número total de preguntas, según dimensiones analizadas para el cálculo de la puntuación de transparencia en las universidades analizadas

\begin{tabular}{|c|c|c|c|}
\hline Dimensión & Indicador & $\begin{array}{c}\text { Subtotal preguntas o } \\
\text { parámetros }\end{array}$ & Total preguntas o parámetros \\
\hline Transparencia formal & $\begin{array}{l}\text { Micronavegación } \\
\text { Macronavegación }\end{array}$ & $\begin{array}{c}47 \\
9\end{array}$ & 56 \\
\hline Transparencia sustantiva & & & 36 \\
\hline \multicolumn{3}{|l|}{ Preguntas totales } & 92 \\
\hline
\end{tabular}

dicha información. Intenta adaptar la Constitución colombiana de 1991 a los estándares internacionales actuales sobre transparencia y rendición de cuentas por medio de 33 artículos y de disposiciones coactivas para todas las ramas del poder público colombiano, y para todas las personas y entidades privadas que cumplan funciones o servicio público y actividades políticas. Se respetan los procedimientos legales de solicitud de acceso a información, la protección de los datos reservados (por un máximo de 15 años se podrá mantener este tipo de información clasificada) y las garantías de los derechos de los grupos poblacionales minoritarios, desde una serie de principios que se recogen en las tablas elaboradas para la medición de los indicadores de transparencia (tablas 1 y 2) del apartado de metodología. Explicamos brevemente los conceptos de:

- micronavegación: estudia la calidad de la web en sí misma;

- macronavegación: determina el grado de visibilidad y de luminosidad de la web y su capacidad de autodescribirse.

Ambos aspectos son muy relevantes para la localización de la universidad por los robots de búsqueda y el posicionamiento.

\section{Metodología}

Este trabajo busca estimar el nivel de transparencia de las instituciones de educación superior en Colombia (universidades, instituciones universitarias y escuelas tecnológicas), desde la publicación de la Ley 1712/2014 de transparencia y derecho de acceso a la información en dicho país.

El estudio toma como base metodológica el trabajo realizado en 2014 por el grupo de investigación Transparencia, Buena Gobernanza y Comunicación, de la Universidad Complutense de Madrid, en el que se aplicó un análisis directo de las webs institucionales a través de un instrumento creado ad hoc. En él se estimaban un conjunto de categorías para la medición de:

- la transparencia formal, relacionada con la idoneidad de la información expuesta en los portales web institucionales;

- la transparencia sustantiva, que alude al conjunto de procedimientos y documentos exigidos por la Ley de Transparencia en Colombia (Ley 1712/2014).

El objetivo final persigue brindar la información necesaria para conocer el funcionamiento de cada institución educativa desde estos escenarios digitales.

Puesto que el instrumento aplicado en España tomó como base el marco legal vigente en dicho país sobre la transparencia (Ley 19/2013, España, 2013), la investigación realizada en Colombia lo adaptó y ajustó al marco legal correspondiente, la Ley 1712/2014. El instrumento aplicado en Colombia consta de 92 preguntas o parámetros, que sirven para la medición de los indicadores que recogen las tablas 1 y 2.

Tabla 2. Principales dimensiones y parámetros de análisis tomados para la medición de la transparencia en las universidades (2015)

\begin{tabular}{|c|c|c|}
\hline Tipo de transparencia & Dimensión de análisis & Parámetros de medición \\
\hline \multirow[t]{2}{*}{ Formal } & Micronavegación & $\begin{array}{l}\text { Referidos al portal institucional } \\
\text { - } \quad \text { Calidad y volumen de la información (relevancia, registro total y orden de la información } \\
\text { - } \quad \text { Legpuesta) } \\
\text { - } \quad \text { Navegación y representación de la información } \\
\text { - } \quad \text { Recuperabilidad de la información a través de las bases de datos } \\
\text { - } \quad \text { Interactividad y computabilidad (vías de comunicación existentes y efectividad e interactividad) } \\
\text { - } \quad \text { Descarga de la información en enlaces (existencia de enlaces activos) } \\
\text { - } \quad \text { Servicios adicionales (software u otros productos alternativos que la Universidad pueda } \\
\text { proporcionar para facilitar el acceso a información) }\end{array}$ \\
\hline & Macronavegación & $\begin{array}{l}\text { Visibilidad y luminosidad (presencia y funcionamiento de enlaces externos y criterios claros de } \\
\text { selección de éstos; además de indexación del portal en motores de búsqueda) } \\
\text { Auto-descripción de la web (presencia de títulos y meta-etiquetas en las páginas del portal) }\end{array}$ \\
\hline Sustantiva & & $\begin{array}{l}\text { Existencia de portal de transparencia } \\
\text { Portal web responde a criterios jerárquicos internos } \\
\text { Datos institucionales que figuran en el portal web } \\
\text { Titularidad de la información expuesta } \\
\text { Acceso a documentos solicitados por terceros } \\
\text { Exposición clara de plazos de respuestas a solicitudes de información } \\
\text { Procedimientos de acceso a información } \\
\text { Exposición de resoluciones dadas a solicitudes de acceso } \\
\text { Exposición de sanciones a funcionarios que no faciliten acceso a documentos solicitados } \\
\text { Coste de acceso a documentos públicos de la institución analizada }\end{array}$ \\
\hline
\end{tabular}


Para este estudio se tuvo en cuenta el índice creado desde la Universidad Complutense de Madrid porque:

- es un índice específicamente diseñado para universidades y no para instituciones públicas en general, cualitativamente muy diferentes entre sí;

- está centrado en la transparencia y práctica efectiva del derecho de acceso a la información institucional desde este tipo de entidades en internet.

En cambio, otros índices orientados a la medición de transparencia (por ej., el promovido por la Corporación Transparencia por Colombia), no se centran en exclusiva en la dimensión de análisis propuesta en este trabajo, sino también en otros aspectos como las acciones de control y sanción, la capacidad institucional para el cumplimiento de las normas y estándares de gestión, y la aplicación de políticas, procedimientos y decisiones internas.

http://indicedetransparencia.org.co/ITN/EducacionSuperior

Se analizaron 197 instituciones de educación superior ${ }^{1}: 83$ universidades y 114 instituciones universitarias o escuelas tecnológicas, públicas y privadas, registradas en el Sistema Nacional de Información de Educación Superior (Snies) del Ministerio de Educación Nacional de Colombia en 2015. https://snies.mineducacion.gov.co/consultasnies/institucion

La medición se hizo en dos momentos:

- primer semestre de 2015 (mayo), al año de haberse implementado la Ley 1712/2014;

- primer semestre de 2016 (mayo), dos años después de haber sido publicada la Ley.

De esta forma se consigue tener dos medidas temporales que ayudan a estimar el nivel de aplicación de la Ley en estas instituciones.

El cálculo de la puntuación total de transparencia $(T)$ de cada una de las universidades analizadas resulta del sumatorio de la transparencia formal (TF) y la transparencia sustantiva (TS), ambas estimadas con un factor de ponderación de 0,6 y 0,4 , respectivamente:

$$
\mathrm{T}=\left(\mathrm{TF}^{*} 0,6\right)+\left(\mathrm{TS}^{*} 0,4\right)
$$

El coeficiente de ponderación de la TF y TS se hizo manteniendo el argumento expuesto en el estudio español de referencia, que otorgaba mayor peso a los aspectos formales que a los sustantivos. Esto se debió a que Ley de Transparencia aplicada en Colombia estimó diferentes períodos de entrada en vigor: en el caso de los entes de carácter nacional, a los 6 meses de publicación (septiembre de 2014) y a las entidades territoriales (locales o regionales), al año (marzo de 2015). Marca pues un contexto de transitoriedad de la aplicación de dicha Ley en el país durante el período de estudio. El trabajo sirve de medida inicial y reconocimiento de las fortalezas y debilidades que presentan las universidades públicas en la aplicación de esta norma. En el caso de las universidades privadas, pese al carácter no vinculante de la ley, se revisa su implementación.

El cálculo de la transparencia formal y sustantiva se hizo teniendo en cuenta el siguiente procedimiento:

- puntuación relativa de las dimensiones de transparencia (formal, micronavegación y macronavegación, y sustantiva) consideradas (100/número total de preguntas o indicadores tenidos en consideración en cada caso);

- sumatorio de los parámetros o preguntas de cada una de las dimensiones de transparencia tenidas en cuenta, identificadas favorablemente en cada uno de los portales institucionales analizados;

- multiplicación de la puntuación de las dimensiones de transparencia analizadas (transparencia formal y sustantiva), por el número total de parámetros identificados favorablemente.

El cálculo de la transparencia formal (TF) se hizo aplicando un coeficiente de ponderación del 0,5 a cada una de las puntuaciones generales obtenidas en la dimensión de micronavegación (MicNav) y macronavegación (MacNav). Es igualmente relevante la calidad de la información y su visibilidad:

$$
\mathrm{TF}=\left(\mathrm{MiCNav}^{*} 0,5\right)+\left(\mathrm{MacNav}^{*} 0,5\right)
$$

El valor total de la puntuación de la transferencia se hizo sobre 100 puntos: 60 en el caso de la transparencia formal y 40 en la transparencia sustantiva. Estos valores son indicadores de una transparencia total en cada caso analizado. Con el fin de manejar mejor los resultados para la construcción de un ranking de transparencia de las 84 universidades analizadas, se estandarizaron las puntuaciones obtenidas en 4 categorías o niveles (tabla 3 ).

\section{Resultados}

\subsection{Transparencia formal}

El nivel de desarrollo de los indicadores considerados en este apartado permite ubicar a todas las instituciones de educación superior en los cuartiles inferiores de estandarización empleados (tabla 3) con una puntuación promedio general (durante todo el estudio) en el Q2 ( $\mu$-TF=27,25/60 puntos) y la totalidad de las instituciones analizadas en dicho cuartil.

Las instituciones de educación superior que más han avanzado en el fortalecimiento de los indicadores formales según la medición de 2015 y 2016 son:

- Universidad Simón Bolívar;

- Universidad San Buenaventura;

- Fundación Universitaria Comfenalco de Santander;

- Fundación Universitaria Colombo Internacional (Unicolombo);

- Universidad Tecnológica de Bolívar.

Todas ellas obtuvieron más de $14 / 60$ puntos entre la medición hecha en 2015 versus la realizada en 2016 en este indicador.

Tabla 3. Niveles de estandarización de puntuaciones de transparencias calculados.

\begin{tabular}{|l|l|}
\hline \multicolumn{1}{|c|}{ Nivel } & \multicolumn{1}{c|}{ Valores } \\
\hline Q4 (alto) & 75-100\% de los puntos máximos posibles \\
\hline Q3 (medio) & $50-74 \%$ de los puntos máximos posibles \\
\hline Q2 (bajo) & 25-49\% de los puntos máximos posibles \\
\hline Q1 (muy bajo) & 0-24\% de los puntos máximos posibles \\
\hline
\end{tabular}


Los datos permiten ver que el conjunto de las instituciones analizadas retrocedió 3,17 puntos (equivalente a 6,2\% de deterioro) en el indicador transparencia formal, una vez cumplidos los dos años de vigencia de la Ley aplicada en Colombia. Este resultado muestra un entorno de desarrollo de portales web con carencias en los siguientes parámetros de medición de la dimensión micro y macronavegación de la transparencia formal:

- calidad y volumen de la información expuesta en estos espacios;

- legibilidad y ergonomía del diseño web aplicado;

- navegación y representación de la información publicada;

- recuperabilidad de la información;

- interactividad y computabilidad entre institución y usuarios en sus webs institucionales;

- publicación de servicios de descarga de información y servicios adicionales;

- visibilidad, luminosidad y auto-descripción de los espacios institucionales estudiados.

El promedio de puntuación del nivel de transparencia formal es mayor en el caso de las instituciones públicas ( $\mu$-TF=
31,79/60 puntos, equivalente al nivel Q3 o nivel medio de transparencia formal), en comparación con las privadas ( $\mu$ $\mathrm{TF}=28,64 / 60$ puntos, equivalente al nivel Q2 o nivel bajo de transparencia formal).

El movimiento open beneficia a las organizaciones para la resolución de problemas sociales y el aumento de la productividad en sectores profesionales de las comunicaciones

Las universidades tuvieron un mejor comportamiento promedio en el avance de los niveles de transparencia formal ( $\mu$-TF $=30,42 / 60$ puntos, equivalente al nivel Q3 o nivel medio), en contraste con las escuelas tecnológicas, cuyo nivel de transparencia promedio se situó en los 28,75/60 puntos (nivel Q2 o nivel bajo).

La tabla 4 muestra el top 20 de las instituciones de educación superior que contaron con puntuaciones promediadas

Tabla 4. Top 20 de instituciones de educación superior, según puntuación de transparencia formal.

\begin{tabular}{|c|c|c|c|c|c|c|}
\hline $\begin{array}{l}\text { Instituciones de educación } \\
\text { superior }\end{array}$ & Tipo de institución & Carácter & $\begin{array}{c}\text { Transparencia } \\
\text { formal } 2015\end{array}$ & $\begin{array}{c}\text { Transparencia } \\
\text { formal } 2016\end{array}$ & $\begin{array}{l}\text { Transparencia } \\
\text { formal prome- } \\
\text { diada (total) }\end{array}$ & $\begin{array}{c}\text { Diferencial } \\
\text { transparencia } \\
\text { formal } 2016 \\
\text { versus } 2015\end{array}$ \\
\hline Escuela de Ingenieros Militares & $\begin{array}{l}\text { Institución universitaria/ } \\
\text { Escuela tecnológica }\end{array}$ & Pública & $39,64 / 60$ & $40,55 / 60$ & $40,10 / 60$ & 0,90 \\
\hline $\begin{array}{l}\text { Corporación Universitaria Lati- } \\
\text { noamericana - CUL }\end{array}$ & $\begin{array}{l}\text { Institución universitaria/ } \\
\text { Escuela tecnológica }\end{array}$ & Privada & $39,86 / 60$ & $38,54 / 60$ & $39,20 / 60$ & $-1,32$ \\
\hline Universidad de Cartagena & Universidad & Pública & $37,31 / 60$ & $40,10 / 60$ & $38,71 / 60$ & 2,79 \\
\hline Universidad de Caldas & Universidad & Pública & $37,83 / 60$ & $38,63 / 60$ & $38,23 / 60$ & 0,80 \\
\hline $\begin{array}{l}\text { Corporación Escuela Tecnológica } \\
\text { del Oriente }\end{array}$ & $\begin{array}{l}\text { Institución universitaria/ } \\
\text { Escuela tecnológica }\end{array}$ & Privada & $40,89 / 60$ & $33,89 / 60$ & $37,39 / 60$ & $-6,99$ \\
\hline Universidad de Nariño & Universidad & Pública & $38,60 / 60$ & $36,05 / 60$ & $37,32 / 60$ & $-2,56$ \\
\hline Universidad de Córdoba & Universidad & Pública & $31,55 / 60$ & $42,60 / 60$ & $37,08 / 60$ & 11,05 \\
\hline Tecnológico de Antioquia & $\begin{array}{l}\text { Institución universitaria/ } \\
\text { Escuela tecnológica }\end{array}$ & Pública & $36,58 / 60$ & $37,43 / 60$ & $37,01 / 60$ & 0,85 \\
\hline $\begin{array}{l}\text { Universidad Autónoma de } \\
\text { Occidente }\end{array}$ & Universidad & Privada & $37,34 / 60$ & $36,50 / 60$ & $36,92 / 60$ & $-0,84$ \\
\hline Universidad de Sucre & Universidad & Pública & $38,39 / 60$ & $35,35 / 60$ & $36,87 / 60$ & $-3,04$ \\
\hline Universidad ECCI & Universidad & Privada & $37,52 / 60$ & $35,56 / 60$ & $36,54 / 60$ & $-1,95$ \\
\hline $\begin{array}{l}\text { Universidad de Cundinamarca } \\
\text { - UDEC }\end{array}$ & Universidad & Pública & $38,16 / 60$ & $34,32 / 60$ & $36,24 / 60$ & $-3,83$ \\
\hline Universidad del Pacifico & Universidad & Pública & $35,19 / 60$ & $37,27 / 60$ & $36,23 / 60$ & 2,08 \\
\hline Escuela Nacional del Deporte & $\begin{array}{l}\text { Institución universitaria/ } \\
\text { Escuela tecnológica }\end{array}$ & Pública & $37,13 / 60$ & $34,92 / 60$ & $36,03 / 60$ & $-2,22$ \\
\hline Escuela Superior de Guerra & $\begin{array}{l}\text { Institución universitaria/ } \\
\text { Escuela tecnológica }\end{array}$ & Pública & $37,31 / 60$ & $34,74 / 60$ & $36,03 / 60$ & $-2,57$ \\
\hline Universidad de la Guajira & Universidad & Pública & $35,89 / 60$ & $36,14 / 60$ & $36,02 / 60$ & 0,24 \\
\hline Universidad de la Salle & Universidad & Privada & $38,48 / 60$ & $33,40 / 60$ & $35,94 / 60$ & $-5,09$ \\
\hline $\begin{array}{l}\text { Corporación Universitaria Refor- } \\
\text { mada-CUR }\end{array}$ & $\begin{array}{l}\text { Institución universitaria/ } \\
\text { Escuela tecnológica }\end{array}$ & Privada & $36,33 / 60$ & $35,43 / 60$ & $35,88 / 60$ & $-0,90$ \\
\hline Universidad del Cauca & Universidad & Pública & $36,90 / 60$ & $34,79 / 60$ & $35,84 / 60$ & $-2,10$ \\
\hline Universidad del Norte & Universidad & Privada & $33,96 / 60$ & $37,73 / 60$ & $35,84 / 60$ & 3,77 \\
\hline \multicolumn{7}{|c|}{ Diferencial promedio total: $-0,55$} \\
\hline
\end{tabular}


Tabla 5. Top 20 de instituciones de educación superior, según puntuación de transparencia sustantiva

\begin{tabular}{|c|c|c|c|c|c|c|}
\hline $\begin{array}{l}\text { Institución de educación } \\
\text { superior }\end{array}$ & Tipo de institución & Carácter & $\begin{array}{c}\text { Transparencia } \\
\text { sustantiva } \\
2015\end{array}$ & $\begin{array}{c}\text { Transparencia } \\
\text { sustantiva } \\
2016\end{array}$ & $\begin{array}{l}\text { Transparencia } \\
\text { sustantiva } \\
\text { promedio }\end{array}$ & $\begin{array}{c}\text { Diferencial } \\
\text { transparencia } \\
\text { sustantiva } 2016 \\
\text { versus } 2015\end{array}$ \\
\hline $\begin{array}{l}\text { Universidad Industrial de San- } \\
\text { tander }\end{array}$ & Universidad & Pública & $11,00 / 40$ & $34,44 / 40$ & $22,72 / 40$ & 23,44 \\
\hline Universidad de Córdoba & Universidad & Pública & $9,65 / 40$ & $30,00 / 40$ & $19,83 / 40$ & 20,35 \\
\hline $\begin{array}{l}\text { Escuela Tecnológica Instituto } \\
\text { Técnico Central }\end{array}$ & $\begin{array}{l}\text { Institución universitaria/ } \\
\text { Escuela tecnológica }\end{array}$ & Pública & $11,76 / 40$ & $26,67 / 40$ & $19,21 / 40$ & 14,91 \\
\hline $\begin{array}{l}\text { Escuela de Postgrados de la Fuer- } \\
\text { za Aérea Colombiana Capitán } \\
\text { José Edmundo Sandoval - EPFAC }\end{array}$ & $\begin{array}{l}\text { Institución universitaria/ } \\
\text { Escuela tecnológica }\end{array}$ & Pública & $7,73 / 40$ & $30,00 / 40$ & $18,87 / 40$ & 22,27 \\
\hline Universidad de Cartagena & Universidad & Pública & $10,41 / 40$ & $26,67 / 40$ & $18,54 / 40$ & 16,25 \\
\hline Universidad de Antioquia & Universidad & Pública & $6,98 / 40$ & $30,00 / 40$ & $18,49 / 40$ & 23,02 \\
\hline Universidad Mariana & Universidad & Privada & $9,77 / 40$ & $26,67 / 40$ & $18,22 / 40$ & 16,90 \\
\hline Universidad la Gran Colombia & Universidad & Privada & $9,56 / 40$ & $26,67 / 40$ & $18,11 / 40$ & 17,11 \\
\hline Escuela Superior de Guerra & $\begin{array}{l}\text { Institución universitaria/ } \\
\text { Escuela tecnológica }\end{array}$ & Pública & $10,41 / 40$ & $25,56 / 40$ & $17,98 / 40$ & 15,15 \\
\hline $\begin{array}{l}\text { Universidad-Colegio Mayor de } \\
\text { Cundinamarca }\end{array}$ & Universidad & Pública & $8,03 / 40$ & $27,78 / 40$ & $17,91 / 40$ & 19,74 \\
\hline Universidad de Caldas & Universidad & Pública & $8,43 / 40$ & $26,67 / 40$ & $17,55 / 40$ & 18,24 \\
\hline Colegio Mayor del Cauca & $\begin{array}{l}\text { Institución universitaria/ } \\
\text { Escuela tecnológica }\end{array}$ & Privada & $6,53 / 40$ & $27,78 / 40$ & $17,15 / 40$ & 21,25 \\
\hline $\begin{array}{l}\text { Politécnico Colombiano Jaime } \\
\text { Isaza Cadavid }\end{array}$ & $\begin{array}{l}\text { Institución universitaria/ } \\
\text { Escuela tecnológica }\end{array}$ & Pública & $12,00 / 40$ & $22,22 / 40$ & $17,11 / 40$ & 10,22 \\
\hline Universidad Libre & Universidad & Privada & $7,46 / 40$ & $26,67 / 40$ & $17,06 / 40$ & 19,21 \\
\hline Universidad ICESI & Universidad & Privada & $10,76 / 40$ & $22,22 / 40$ & $16,49 / 40$ & 11,46 \\
\hline Escuela Nacional del Deporte & $\begin{array}{l}\text { Institución universitaria/ } \\
\text { Escuela tecnológica }\end{array}$ & Pública & $7,73 / 40$ & $24,44 / 40$ & $16,09 / 40$ & 16,71 \\
\hline $\begin{array}{l}\text { Universidad del Magdalena- } \\
\text { UNIMAGDALENA }\end{array}$ & Universidad & Pública & $8,43 / 40$ & $23,33 / 40$ & $15,88 / 40$ & 14,91 \\
\hline $\begin{array}{l}\text { Corporación Escuela Tecnológica } \\
\text { del Oriente }\end{array}$ & $\begin{array}{l}\text { Institución universitaria/ } \\
\text { Escuela tecnológica }\end{array}$ & Privada & $11,49 / 40$ & $20,00 / 40$ & $15,74 / 40$ & 8,51 \\
\hline $\begin{array}{l}\text { Universidad Nacional Abierta y a } \\
\text { Distancia UNAD }\end{array}$ & Universidad & Pública & $11,28 / 40$ & $20,00 / 40$ & $15,64 / 40$ & 8,72 \\
\hline Conservatorio del Tolima & $\begin{array}{l}\text { Institución universitaria/ } \\
\text { Escuela tecnológica }\end{array}$ & Privada & $6,68 / 40$ & $24,44 / 40$ & $15,56 / 40$ & 17,76 \\
\hline \multicolumn{7}{|c|}{ Diferencial promedio total: 16,81} \\
\hline
\end{tabular}

mayores en cuanto a los criterios de micro y macronavegación de sus portales:

- Las instituciones de educación superior con mayores puntuaciones en transparencia formal fueron las que presentaron un diferencial promedio menor (-0,55 puntos, entre las puntuaciones obtenidas en 2016 versus las de 2015, equivalente apenas a $0,91 \%$ de retroceso entre las mediciones realizadas), a diferencia de lo observado en la muestra total: 197 instituciones (-3,17/60 puntos prome- dio de variación entre los datos obtenidos en 2016 versus los recabados en 2015).

- Salvo casos como el de la Universidad de Córdoba, donde se aprecia una evolución positiva significativa en 2016 frente a lo observado en 2015 en transparencia formal (11,05 puntos de mejora, equivalente a $18,41 \%$ de avance en la puntuación máxima posible de obtenerse en este apartado), las instituciones con mejores puntuaciones en este apartado ayudan a reafirmar la poca o inexistente 
evidencia de mejora en los parámetros de medición de las dimensiones micro y macronavegación relacionados con la transparencia formal de sus webs institucionales. Se dibuja un panorama de instituciones donde la Ley 1712/2014 no parece haber contado con medidas institucionales que ayuden a su aplicación.

En el top de instituciones de educación superior que lideran la transparencia formal en Colombia predominan las entidades de tipo público y las universidades.

\section{La transformación de la cultura digital en las universidades incluye la forma en que se muestra la información en los ca- nales web 2.0 institucionales}

\subsection{Transparencia sustantiva}

El escenario resulta más diverso y negativo según la ubicación en cuartiles de estandarización de los niveles de transparencia: apenas el 53,29\% del total de las instituciones se ubicaron en un Q2 (nivel bajo), y el 46,19\% se encontró en el nivel Q1 o nivel muy bajo de transparencia sustantiva. Sólo una institución (la Universidad Industrial de Santander con 22,72/40 puntos posibles) se situó en el nivel Q3 o nivel medio de transparencia sustantiva. Por tanto, estamos ante el tipo de transparencia que más debilidades presentó tras dos años de la entrada en vigor de la Ley de Transparencia.

Pese al nivel de la transparencia sustantiva observada en los portales de las instituciones de educación superior colombiana, el conjunto de las 197 organizaciones estudiadas presentó un avance positivo promedio de 5,48 puntos en 2016, en comparación con la medición inicial hecha en 2015. Esto equivale a una mejora del $13,7 \%$ de la calificación máxima posible en este apartado; el $29,44 \%$ de los casos estudiados (58 instituciones de educación superior) presentaron un aumento significativo del nivel de transparencia sustantiva equivalente o superior al 20\% (mínimo 8 puntos de crecimiento entre 2015 y 2016). El dato, aun siendo precario, señala mejorías en estas webs: la exposición y acceso a datos institucionales e información de la titularidad de la información expuesta, exposición de resoluciones y sanciones y la garantía de un mayor número de mecanismos que faciliten el acceso a documentos solicitados por terceros en sus portales web, entre otras.

Al comparar la transparencia sustantiva observada en las universidades públicas y las privadas, y entre las universidades y las instituciones universitarias o escuelas tecnológicas, observamos que:

- las instituciones de educación superior públicas presentaron una puntuación promedio de $13,17 / 40$ puntos, equivalente al nivel Q2 o bajo de transparencia sustantiva;

- las instituciones de educación superior privadas obtuvieron una puntuación promedio de 9,96/40 puntos, ubicándoles en el nivel Q1 o nivel muy bajo de transparencia sustantiva.

En el caso de las universidades frente a las instituciones universitarias o escuelas tecnológicas, las puntuaciones pro- medio observados no resultaron tan diferentes $(11,46 / 40$ puntos y $10,35 / 40$ puntos, respectivamente): ubican a los miembros de ambos grupos en el mismo nivel de estandarización expuesto en la tabla 3 (Q2 o nivel bajo de transparencia sustantiva).

En cuanto a las instituciones de educación superior que lideraron los niveles de transparencia sustantivos observados durante las mediciones hechas en 2015 y 2016, la tabla 5 muestra cómo:

- Las instituciones de educación superior, con mayores niveles de transparencia sustantiva fueron las que presentaron un crecimiento promedio de 16,81 puntos; mostraron además un crecimiento en todos los casos de más del $100 \%$ de las puntuaciones obtenidas por estas entidades durante la primera medición.

- En el top de instituciones de educación superior que lideran la transparencia sustantiva en Colombia predominan las entidades de tipo público frente a las privadas. También hay mayor presencia de instituciones universitarias que de escuelas tecnológicas.

\subsection{Nivel de transparencia total}

La puntuación promedio $(\mu$ ) fue del 40,27/100 puntos. La media observada muestra un contexto en el que la mayoría (87,30\%, equivalente a 172 instituciones) de las instituciones de educación superior analizadas se ubican en el nivel Q2 o nivel bajo de transparencia. Sólo el 10,65\% (21 instituciones) se encuentran en el nivel Q3 o nivel medio y el 2,03\% (4 instituciones) en el nivel Q1 o nivel muy bajo.

Las instituciones de educación situadas en el Q3 de transparencia son en su mayoría instituciones universitarias o escuelas técnicas (55\%) y de carácter público (80\%); mientras que las que se ubican en el nivel Q1, son en su totalidad instituciones universitarias o escuelas tecnológicas privadas; y las que se sitúan en el nivel bajo o Q2 se caracterizan por ser en su mayoría instituciones privadas $(79,06 \%)$ y escuelas tecnológicas $(57,55 \%)$.

\section{El impacto transformador ocasionado por la web 2.0 en las instituciones re- quiere la implantación de nuevas diná- micas de promoción de la cibercultura y la transparencia}

En el caso de las universidades, las públicas presentaron un mayor nivel promedio de transparencia $(\mu-\mathrm{TF}=42,8 / 100$ puntos), y un nivel diferencial promedio positivo entre los datos obtenidos en 2015 frente a los de 2016 de 8,25 puntos; mientras que las privadas obtuvieron una puntuación de transparencia promedio ( $\mu$-TF) de $39,32 / 100$ puntos y un nivel diferencial promedio positivo de 2,99 puntos. Si bien en ambos casos se ubican en el nivel Q2 o bajo de transparencia, sí se aprecia una mejor puntuación de las entidades públicas frente a las privadas según el marco de aplicación de la Ley examinada y en estrategias para su puesta en marcha en portales web institucionales, desde la primera medición (2015) hasta la última (2016). 
Tabla 6. Top 20 de instituciones de educación superior, según puntuación de transparencia total.

\begin{tabular}{|c|c|c|c|c|c|c|}
\hline $\begin{array}{l}\text { Institución de educación } \\
\text { superior }\end{array}$ & Tipo de institución & Carácter & $\begin{array}{l}\text { Transpa- } \\
\text { rencia total } \\
2015\end{array}$ & $\begin{array}{l}\text { Transpa- } \\
\text { rencia total } \\
2016\end{array}$ & $\begin{array}{l}\text { Transpa- } \\
\text { rencia total } \\
\text { promedio }\end{array}$ & $\begin{array}{l}\text { Diferencial trans- } \\
\text { parencia total } \\
2016 \text { versus } 2015\end{array}$ \\
\hline Universidad de Cartagena & Universidad & Pública & $47,73 / 100$ & $66,77 / 100$ & $57,25 / 100$ & 19,04 \\
\hline Universidad de Córdoba & Universidad & Pública & $41,20 / 100$ & $72,60 / 100$ & $56,90 / 100$ & 31,40 \\
\hline Universidad de Caldas & Universidad & Pública & $46,25 / 100$ & $65,30 / 100$ & $55,78 / 100$ & 19,04 \\
\hline $\begin{array}{l}\text { Escuela de Postgrado de la Fuer- } \\
\text { za Aérea Colombiana Capitán } \\
\text { José Edmundo Sandoval - EPFAC }\end{array}$ & $\begin{array}{l}\text { Institución universitaria/ } \\
\text { Escuela tecnológica }\end{array}$ & Pública & $44,87 / 100$ & $63,47 / 100$ & $54,17 / 100$ & 18,61 \\
\hline Escuela Superior de Guerra & $\begin{array}{l}\text { Institución universitaria/ } \\
\text { Escuela tecnológica }\end{array}$ & Pública & $47,72 / 100$ & $60,30 / 100$ & $54,01 / 100$ & 12,58 \\
\hline $\begin{array}{l}\text { Escuela Tecnológica Instituto } \\
\text { Técnico Central }\end{array}$ & $\begin{array}{l}\text { Institución universitaria/ } \\
\text { Escuela tecnológica }\end{array}$ & Pública & $52,92 / 100$ & $54,00 / 100$ & $53,46 / 100$ & 1,08 \\
\hline $\begin{array}{l}\text { Universidad Industrial de San- } \\
\text { tander }\end{array}$ & Universidad & Pública & $46,40 / 100$ & $60,46 / 100$ & $53,43 / 100$ & 14,05 \\
\hline $\begin{array}{l}\text { Corporación Escuela Tecnológica } \\
\text { del Oriente }\end{array}$ & $\begin{array}{l}\text { Institución universitaria/ } \\
\text { Escuela tecnológica }\end{array}$ & Privada & $52,37 / 100$ & $53,89 / 100$ & $53,13 / 100$ & 1,52 \\
\hline Universidad de Antioquia & Universidad & Pública & $43,37 / 100$ & $62,21 / 100$ & $52,79 / 100$ & 18,84 \\
\hline $\begin{array}{l}\text { Politécnico Colombiano Jaime } \\
\text { Isaza Cadavid }\end{array}$ & $\begin{array}{l}\text { Institución universitaria/ } \\
\text { Escuela tecnológica }\end{array}$ & Pública & $48,39 / 100$ & $55,94 / 100$ & $52,17 / 100$ & 7,55 \\
\hline Escuela Nacional del Deporte & $\begin{array}{l}\text { Institución universitaria/ } \\
\text { Escuela tecnológica }\end{array}$ & Pública & $44,87 / 100$ & $59,36 / 100$ & $52,11 / 100$ & 14,49 \\
\hline Tecnológico de Antioquia & $\begin{array}{l}\text { Institución universitaria/ } \\
\text { Escuela tecnológica }\end{array}$ & Pública & $48,76 / 100$ & $54,10 / 100$ & $51,43 / 100$ & 5,34 \\
\hline Universidad ICESI & Universidad & Privada & $45,93 / 100$ & $55,82 / 100$ & $50,88 / 100$ & 9,89 \\
\hline Universidad de Nariño & Universidad & Pública & $47,81 / 100$ & $53,82 / 100$ & $50,82 / 100$ & 6,02 \\
\hline Escuela de Ingenieros Militares & $\begin{array}{l}\text { Institución universitaria/ } \\
\text { Escuela tecnológica }\end{array}$ & Pública & $49,89 / 100$ & $51,66 / 100$ & $50,77 / 100$ & 1,77 \\
\hline $\begin{array}{l}\text { Corporación Universitaria de } \\
\text { Ciencias Empresariales, Educa- } \\
\text { ción y Salud - CORSALUD }\end{array}$ & $\begin{array}{l}\text { Institución universitaria/ } \\
\text { Escuela tecnológica }\end{array}$ & Privada & $47,68 / 100$ & $53,56 / 100$ & $50,62 / 100$ & 5,88 \\
\hline Universidad del Norte & Universidad & Privada & $43,32 / 100$ & $57,73 / 100$ & $50,52 / 100$ & 14,41 \\
\hline $\begin{array}{l}\text { Universidad Del Magdalena - } \\
\text { UNIMAGDALENA }\end{array}$ & Universidad & Pública & $46,25 / 100$ & $54,67 / 100$ & $50,46 / 100$ & 8,41 \\
\hline $\begin{array}{l}\text { Institución Universitaria Antonio } \\
\text { José Camacho }\end{array}$ & $\begin{array}{l}\text { Institución universitaria/ } \\
\text { Escuela tecnológica }\end{array}$ & Pública & $46,44 / 100$ & $54,14 / 100$ & $50,29 / 100$ & 7,70 \\
\hline Universidad del Cauca & Universidad & Pública & $44,39 / 100$ & $55,90 / 100$ & $50,15 / 100$ & 11,51 \\
\hline
\end{tabular}

En lo que se refiere a las escuelas tecnológicas, las públicas presentaron tanto una mayor puntuación promedio a nivel de transparencia ( $\mu$-TF=39,27/100 puntos) como en el diferencial promedio positivo de los datos de 2015 frente a los de 2016 (4,87 puntos). En comparación las privadas alcanzaron un nivel de transparencia total promedio ligeramente inferior a lo observado en el caso anterior ( $\mu$-TF=38,15/100 puntos), y el diferencial promedio entre los datos recabados en 2015 frente a los de 2016 fue ligeramente negativo (-0,56 puntos). Unos datos que muestran cómo este tipo de entidades se sitúan en los últimos puestos de promoción de la transparencia en Colombia, pese a encontrarse, como el resto de entidades analizadas, en el nivel Q2 o bajo desarrollo. A su vez mostraron que en el periodo de estudio las entidades privadas hicieron poca labor por avanzar en el proceso de transparencia exigido legalmente en Colombia.
Los datos sobre los niveles de transparencia ayudan a visualizar un marco institucional donde las instituciones públicas tienen mayor consciencia o interés por incorporar medidas que las privadas. Y esto pese a que la Ley 1712/2014, no sólo está dirigida a las primeras, sino a todas las entidades u organizaciones que ejercen una labor educativa.

Como se aprecia en la tabla 6, el ranking general del top 20 de instituciones de educación superior con mayores puntuaciones de transparencia en Colombia está integrado mayoritariamente (80\%) por entidades públicas. Estas instituciones muestran mayores niveles de avances si tenemos en cuenta los datos de 2015 frente a los de 2016, en los que se obtuvo un diferencial promedio positivo de 11,46 puntos. Es decir, fueron instituciones que no sólo lideraron el escaso avance general de la implementación de la Ley de Transparencia impulsada en Colombia; también las acciones que se 
llevaron a cabo entre la primera medición y la última aplicada en este trabajo dieron un avance promedio mayor que el resto de instituciones analizadas.

\section{Conclusiones}

Después de algo más de tres meses de finalizar el plazo de ejecución efectiva de la Ley 1712/2014 de transparencia en Colombia, y pese a los plazos de aplicación de la normativa, los niveles de desarrollo general de transparencia de los portales web de las universidades colombianas son medios. Este hecho es más crítico con relación a la transparencia sustantiva -es decir, al conjunto de procedimientos y documentos exigidos por la Ley- que a la transparencia formal, relacionada con la macro y micronavegación.

Los datos muestran en las universidades analizadas una cultura e identidad institucional todavía en desarrollo, donde el impacto transformador de la web 2.0, y otros procesos relacionados con la cibercultura -datos abiertos y movimientos open, por citar algunos-, presentan dificultades de aplicación efectiva.

Esta dificultad es más pronunciada en las universidades privadas que en las públicas, lo que subraya la precariedad de la ejecución efectiva de la Ley en las instituciones analizadas.

En los centros analizados el impacto transformador de la web 2.0 presenta dificultades de aplicación efectiva

Con todo, se aprecia un tímido crecimiento de concienciación y voluntad de cambio hacia la transparencia. Este moderado avance en el tiempo se plasma en acciones como el acceso a datos institucionales e información de la titularidad de la información expuesta, la exposición de resoluciones y sanciones, o un mayor número de mecanismos de acceso a documentos solicitados por terceros.

Los datos recabados delinean un contexto general y comparativo de los niveles de transparencia de las universidades colombianas en un marco de tiempo específico, por lo que es aconsejable avanzar en el análisis periódico de estos aspectos para estimar su avance o estancamiento. No olvidemos que son parámetros que inciden en la transformación de la cultura e identidad institucional en un momento de auge de los escenarios digitales actuales.

\section{Notas}

1. No se incluyeron en el estudio el portal de la Universidad Autónoma de Bucaramanga (UNAB); el portal de la Fundación Universitaria Tecnológico Confenalco de Cartagena, ni el portal de la Fundación Universitaria Konrad Adenauer por no haber logrado información total o parcial de estas instituciones, durante las dos fechas de medición de este trabajo.

\section{Referencias}

Anderson, Janna-Quitney; Boyles, Jan-Lauren; Rainie, Lee (2012). The future impact of the internet on higher education: Experts expect more efficient collaborative environ- ments and new grading schemes; they worry about massive online courses, the shift away from on-campus life. Pew Internet \& American Life Project.

http://www.pewinternet.org/files/old-media/Files/ Reports/2012/PIP_Future_of_Higher_Ed.pdf

Bates, Jo (2012). Open data movement redux: tribes and contradictions.

http://goo.gl/Wqbbk

Bernal, Natasha (2012). "Open data, mejor que big data para generar beneficios a empresas". TICbeat, 24 agosto. http://www.ticbeat.com/innovacion/open-data-mejor-bigdata

Connaway, Lynn-Silipigni (2014). “¿Cuál es el sentido de las bibliotecas? Un Ilamamiento para una evaluación centrada en el uso". BiD: textos universitaris de biblioteconomia i documentació, n. 32.

https://doi.org/10.1344/BiD2014.32.4

Downes, Stephen (2001). "Learning objects: Resources for distance education worldwide". The international review of research in open and distributed learning, v. 2, n. 1. http://www.irrodl.org/index.php/irrodl/article/view/32/378

España (2013). "Ley 19/2013, de 9 de diciembre, de transparencia, acceso a la información pública y buen gobierno". $B O E$, n. 295, 10 de diciembre.

https://www.boe.es/buscar/doc.php?id=BOE-A-2013-12887

Farrell, Henry (2012). "Trish, Reiner and the politics of open data". Crooked timber open data, pp. 30-34.

http://crookedtimber.org/2012/07/04/trish-reiner-and-thepolitics-of-open-data

Ferrer-Sapena, Antonia; Sánchez-Pérez, Enrique (2013). "Open data, big data: ¿hacia dónde nos dirigimos?". Anuario ThinkEPI, v. 7, pp. 150-156.

http://eprints.rclis.org/21006/1/150-156-Ferrer-SanchezOpen-data-Big-data.pdf

García-Macho, Ricardo (2010). “El derecho a la información, la publicidad y la transparencia en las relaciones entre la administración, el ciudadano y el público". En: García-Macho, Ricardo (ed.). Derecho administrativo de la información y administración transparente. Madrid: Marcial Pons, pp. 27-48. ISBN: 9788497688222

Gewin, Virginia (2016). "Data sharing: an open mind on open data". Nature, v. 529, n. 7584, pp. 117-119. https://doi.org/10.1038/nj7584-117a

Colombia (2014). Ley 1712 por medio de la cual se crea la Ley de transparencia y del derecho de acceso a la información pública nacional y se dictan otras disposiciones. 6 marzo. https://goo.gl/gbixGg

Hernández-Pérez, Tony; García-Moreno, María-Antonia (2013). "Datos abiertos y repositorios de datos: nuevo reto para los bibliotecarios". El profesional de la información, v. 22, n. 3, pp. 259-263. https://doi.org/10.3145/epi.2013.may.10

Johnson, Steven (2012). "Searching for John Snows". Crooked timber, 27 June. 
http://crookedtimber.org/2012/06/27/searching-for-john-snows

Lara, Tíscar (2009). "El papel de la universidad en la construcción de su identidad digital". Revista de universidad y sociedad del conocimiento, v. 6, n. 1, pp. 15-21.

https://doi.org/10.7238/rusc.v6i1.25

Lathrop, Daniel; Ruma, Laurel (eds.) (2010). Open government. California: O’Reilly Media. ISBN: 9780596804350

Lee, Tom (2012). "Open data: Better politics, winning politics... but still politics". Crooked timber, 7 June.

http://crookedtimber.org/2012/07/06/open-data-betterpolitics-winning-politics-but-still-politics

Manfredi-Sánchez, Juan-Luis (2017). "Horizontes de la información pública". El profesional de la información, v. 26, n. 3, pp. 353-360.

https://doi.org/10.3145/epi.2017.may.01

Mengual-Andrés, Santiago (2013). "Rethinking the role of higher education". Journal of new approaches in educational research, v. 2, n. 1, pp. 1-2.

https://doi.org/10.7821/naer.2.1.1-2

Montoya-Santiago, Carolina (2016). "La transparencia en las universidades públicas españolas, según la Ley 19/2013, de Transparencia, acceso a la información pública y buen gobierno". En: Cousido, Pilar (coord.). Libro blanco sobre gestión de oficinas de transparencia: procedimientos y casos de estudio para universidades públicas. Valencia: Tirant lo Blanch, pp. 33-123. ISBN: 9788490869079

Newman, David (2012). “Open for business: learn to profit by open data". Gartner, 9 March.

http://www.gartner.com/id=1947015

Noveck, Beth (2012). "Open data. The democratic imperative". Crooked timber, 5 July.

http://crookedtimber.org/2012/07/05/open-data-the-democratic-imperative

Prince, Alejandro; Jolías, Lucas (2013). “Open data. Las fuen- tes conceptuales del gobierno abierto". Telos, v. 94, pp. 1-9. https://goo.gl/57MRFe

Rebolledo, Marta; Zamora-Medina, Rocío; Rodríguez-Virgili, Jordi (2017). "Transparency in citizen participation tools and public information: A comparative study of the Spanish councils' websites". El profesional de la información, v. 26, n. 3, pp. 361-369.

https://doi.org/10.3145/epi.2017.may.02

Roig, Rosabel; Mengual-Andrés, Santiago; Suárez-Guerrero, Cristóbal (2014). "Evaluación de la calidad pedagógica de los mooc". Profesorado, v. 18, n. 1, pp. 27-41.

https://recyt.fecyt.es//index.php/profesorado/article/ view/42888/24768

Scassa, Teresa; Campbell, Lisa (2009). "Data protection, privacy and spatial data". En: Devillers, Rodolphe; Goodchild, Helen (ed.). Spatial data quality. CRC Press, pp. 211-219. ISBN: 9781439810125

https://doi.org/10.1201/b10305-24

Sommerman, Kark-Peter (2010). La exigencia de una administración transparente en la perspectiva de los principios de democracia y del estado de derecho. En: García-Macho Ricardo (ed.). Derecho administrativo de la información y administración transparente. Madrid: Marcial Pons, 2010, p. 11-26. ISBN: 9788497688222

Venkatesh, Viswanath; Thong, James; Chan, Frank; Hu, Paul (2016). "Managing citizens' uncertainty in e-government services: The mediating and moderating roles of transparency and trust". Information systems research, v. 27, n.1, pp. 87-111.

https://doi.org/10.1287/isre.2015.0612

Yglesias, Matthew (2012). "Open data journalism". Crooked timber, 28 June.

http://crookedtimber.org/2012/06/28/open-data-journalism

Yu, Harlan; Robinson, David (2012). The new ambiguity of open government. USA: UCLA Law Review.

Si te interesan los

INDIGADORES. EN GIENCIAY TEGNOLOGÍA,

y todọs los temas relacionados con la medición de la ciencia, tales como:

Análisis de citas, Normalización de nombres e instituciones, Impacto de la ciencia en la sociedad, Indicadores, Souciolog de la ciencia, Política científica, Comunicación de la cienát Reviștảs, Bases de datos, Índices

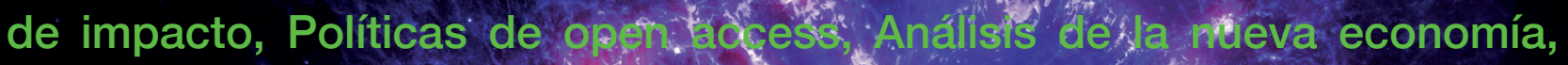
Mujer y ciencia, etc.

Entonces INGY es tu lista. Suscribete en:

http://wWw.rediris.es/list/info/incyt.htmI . 
